\title{
Testing for convergence in left-right ideological positions
}

\author{
ROB EISINGA \\ Department of Social Science Research Methods, University of Nijmegen \\ and
}

\section{PHILIP HANS FRANSES}

Department of Econometrics, Erasmus University Rotterdam

\begin{abstract}
This paper investigates convergence in left-right ideological positions in The Netherlands using cointegration techniques. Our sample consists of 765 weekly observations on those positions as well as on the corresponding political party preference. The time series data display nonstationary patterns in the sense that their means are not constant over time. Therefore, we rely on recently developed techniques in the analysis of multivariate nonstationary time series to study convergence. One of our results is that the ideological positions, when considered relative to a benchmark, can be described by trend-stationary processes. This means that we cannot reject the presence of convergence. Implications of this result are discussed.
\end{abstract}

\section{Introduction}

For decades political scientists have summarized the ideological orientation of voters in Western Europe by their position on a single left-right continuum. This position is considered to reflect a general attitude towards the socioeconomic structure of society: a position on the left indicates a favourable attitude towards changes in the direction of greater (social, political, and economic) equality, while a location on the right side represents opposition to such changes. The terms left and right are thus related to proletarian and bourgeois interests and traditionally these interests have been advanced by main class parties. In modern industrial societies, however, the relevance of class and class conflicts for political parties has declined. In addition, the mainline political parties have changed their character and electoral strategies: they have become 'catch-all' organizations targeting different socio-economic groups. Several political scientists have therefore suggested that the political expression in terms of left-right dimension is losing its relevance for political party preference and that the once considerable left-right distinctions between different groups of voters are gradually disappearing, resulting in an ideological more homogeneous electorate.

Unfortunately, however, there has as yet been no empirical attempt to 
test this hypothesis of convergence in ideological positions, an important reason being the lack of both longitudinal data and an appropriate method to study convergence. This study draws upon 765 independent, national surveys spanning 15 years to examine convergence in the ideological leftright position of voters for six (combinations of) political parties in the Netherlands for the 1978-1992 period. It puts the convergence hypothesis onto a firm basis and its shows that for an appropriate analysis of the data, we need to rely on recently developed econometric techniques in cointegration analysis.

The paper is organized as follows. Section 2 discusses the data used in this study and analyses the univariate time series. Section 3 considers some concepts in cointegration analysis, summarizing the results we use in testing convergence. Section 4 applies the method we propose to the data on leftright position and concluding remarks are offered in Section 5.

\section{The data}

The data are given by NIPO's Omnibus Survey, a weekly survey based on personal interviews of a random probability sample of the Dutch voting age population living in private households, that has been running with a relatively fixed design since the early 1950s. Next to topics that are rotated into the weekly surveys periodically and those that are included on an occasional basis, the surveys also contain a series of standardized questionnaire items. These items include, among others, the respondents' current political party preference and their left-right ideological position. We have extracted these characteristics from all weekly surveys conducted during the past four decades. The survey that first measured left-right position was conducted in 1978. Therefore our analysis is restricted to the 765 weekly surveys - with a total of 571,482 valid responses - conducted between week 1 of 1978 and week 53 of 1992. A detailed guide to this mammoth data file can be found in the documentation of Eisinga and Felling (1992). To measure political party preference the surveys asked respondents how they would vote if an election were held at the day of the interview. The variable has been collapsed into six responses, hereafter labelled $K R_{t}$ (minor right-wing religious parties), $V V D_{t}$ (liberal-conservative party), $C D A_{t}$ (Christian democratic party), $D 66_{t}$ (left-liberal party), $P v d A_{t}$ (social democratic party), and $K L_{t}$ (minor left-wing nonreligious parties). Left-right ideological position was measured by the respondent's self-placement on a 7-point scale, with end points labelled 'left' and 'right', respectively. 


\section{Left-Right Ideological Positions and Political Party Preference}

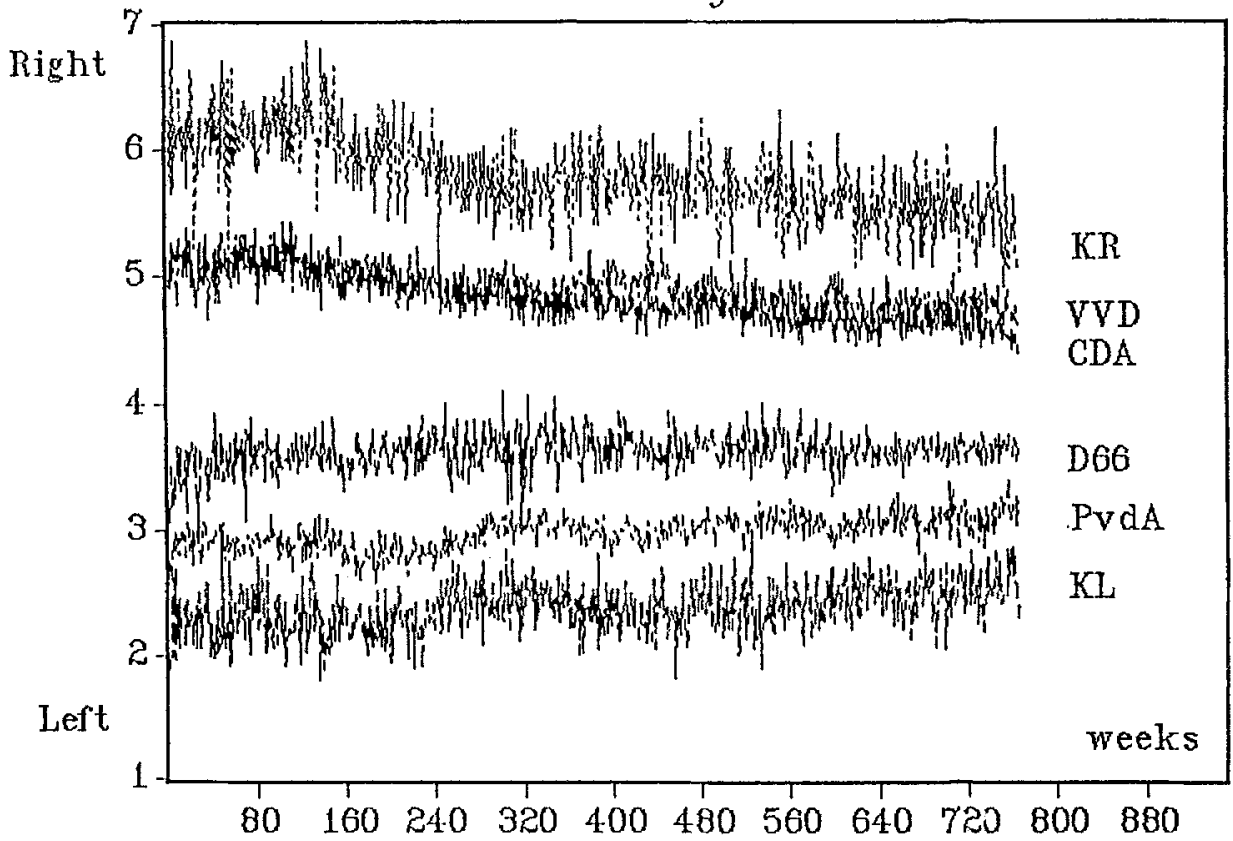

Fig. 1.

\section{Univariate analysis}

To examine the convergence hypothesis the mean values for each of the six groups of voters on the left-right continuum were obtained. The univariate time series with a total of 765 weekly observations are displayed in Figure 1. The graphs or the data reveal that the univariate time series show nonstationary patterns, i.e., there seems to be a trending behaviour. To get a first impression of the data, we have calculated the autocorrelations at lags 1,2 , $3,4,5,10,15$, and 20 . The last three autocorrelations should give an indication of the pace at which the autocorrelations die out. When the values of these correlations die out slowly, we have an indication that the time series should be first order differenced, see Box and Jenkins (1970).

In the first panel of Table 1, we present the estimated autocorrelations for each of the six series. It can be observed that the values remain high, even at 20 lags. Given the graphs in Figure 1, one may conjecture that this long memory in the time series may be caused by a deterministic trend. Hence in the second panel of Table 1, we report the autocorrelations calculated 
Table 1. Autocorrelations for the ideological position series

\begin{tabular}{lllllll}
\hline \multirow{2}{*}{ Autocorrelation at lag } & \multicolumn{2}{l}{ Series } \\
\cline { 2 - 7 } & $C D A$ & $P v d A$ & $V V D$ & $D 66$ & $K L$ & $K R$ \\
\hline 1 & $0.821^{*}$ & $0.607^{*}$ & $0.565^{*}$ & $0.117^{*}$ & $0.265^{*}$ & $0.493^{*}$ \\
2 & $0.821^{*}$ & $0.559^{*}$ & $0.561^{*}$ & $0.140^{*}$ & $0.243^{*}$ & $0.474^{*}$ \\
3 & $0.812^{*}$ & $0.570^{*}$ & $0.548^{*}$ & $0.135^{*}$ & $0.266^{*}$ & $0.486^{*}$ \\
4 & $0.805^{*}$ & $0.580^{*}$ & $0.551^{*}$ & 0.044 & $0.235^{*}$ & $0.476^{*}$ \\
5 & $0.794^{*}$ & $0.558^{*}$ & $0.541^{*}$ & $0.081^{*}$ & $0.251^{*}$ & $0.453^{*}$ \\
10 & $0.777^{*}$ & $0.533^{*}$ & $0.547^{*}$ & $0.177^{*}$ & $0.225^{*}$ & $0.476^{*}$ \\
15 & $0.749^{*}$ & $0.474^{*}$ & $0.502^{*}$ & 0.039 & $0.221^{*}$ & $0.458^{*}$ \\
20 & $0.747^{*}$ & $0.456^{*}$ & $0.493^{*}$ & $0.109^{*}$ & $0.168^{*}$ & $0.413^{*}$
\end{tabular}

After regression on a deterministic trend variable

\begin{tabular}{rllllll}
1 & $0.261^{*}$ & $0.348^{*}$ & $0.192^{*}$ & $0.087^{*}$ & $0.121^{*}$ & $0.108^{*}$ \\
2 & $0.263^{*}$ & $0.273^{*}$ & $0.185^{*}$ & $0.110^{*}$ & $0.096^{*}$ & $0.078^{*}$ \\
3 & $0.236^{*}$ & $0.300^{*}$ & $0.158^{*}$ & $0.106^{*}$ & $0.124^{*}$ & $0.100^{*}$ \\
4 & $0.231^{*}$ & $0.316^{*}$ & $0.167^{*}$ & 0.012 & $0.086^{*}$ & $0.079^{*}$ \\
5 & $0.193^{*}$ & $0.284^{*}$ & $0.150^{*}$ & -0.053 & $0.108^{*}$ & 0.044 \\
10 & $0.205^{*}$ & $0.260^{*}$ & $0.166^{*}$ & $0.154^{*}$ & $0.087^{*}$ & $0.109^{*}$ \\
15 & $0.159^{*}$ & $0.176^{*}$ & $0.092^{*}$ & 0.014 & $0.086^{*}$ & $0.095^{*}$ \\
20 & $0.206^{*}$ & $0.158^{*}$ & $0.098^{*}$ & $0.089^{*}$ & 0.027 & 0.018 \\
\hline
\end{tabular}

* Significant at a 5\% level, since the value exceeds twice the standard error. The approximate standard error is $1 / T^{1 / 2}$, which is about 0.036 here.

after each time series has been regressed on a deterministic trend variable $t=1,2, \ldots, T$. Again, one can observe that the autocorrelations do not die out rapidly, even though their values reduce substantially. In sum, from Table 1 it can be concluded that the inclusion of a deterministic trend variable does not remove the trending pattern completely. In that case, it seems useful to remove the trend using the first order differencing filter $\Delta_{1}$, where $\Delta_{k}$ is defined by $\Delta_{k} Z_{t}=\left(1-B^{k}\right) Z_{t}=Z_{t}-Z_{t-k}$, where $B$ is the familiar backward shift operator, see Box and Jenkins (1970). If exactly the $\Delta_{1}$ filter is needed to remove the nonstationary patterns in a time series, such a variable is called $I(1)$, i.e., integrated of order 1 . If no filter is needed, a variable is $I(0)$.

We now proceed with the construction of univariate time series models for the $\Delta_{1}$ transformed variables. Before we start analyzing multivariate time series processes, it seems useful to study the univariate properties of the individual variables. This construction closely follows the standard guidelines as proposed in Box and Jenkins (1970), and which consists of four steps: identification using the autocorrelation function, estimation, testing, and modification. The model class we consider is the autoregressive-moving average model [ARMA], which for $I(1)$ time series becomes ARIMA. In Table 
Table 2. Adequate univariate models for the ideological position series

\begin{tabular}{lllll}
\hline Variable & Model $^{\mathrm{a}}$ & Estimate of $\theta^{\mathrm{b}}$ & Diagnostics $^{c}$ & \\
\hline$K R_{t}$ & $\operatorname{IMA}(1,1)$ & $-0.931(0.017)$ & $\mathrm{BP}(20)=30.18$ & $\mathrm{JB}=25.91^{*}$ \\
$V V D_{t}$ & $\operatorname{IMA}(1,1)$ & $-0.896(0.018)$ & $\mathrm{BP}(20)=12.75$ & $\mathrm{JB}=4.960$ \\
$C D A_{t}$ & $\operatorname{IMA}(1,1)$ & $-0.889(0.017)$ & $\mathrm{BP}(20)=22.23$ & $\mathrm{JB}=7.118^{*}$ \\
$D 66_{r}$ & ARIMA(7,1,1) & $-0.910(0.017)$ & $\mathrm{BP}(20)=15.11$ & $\mathrm{JB}=23.34^{*}$ \\
$P v d A_{t}$ & $\operatorname{IMA}(1,1)$ & $-0.849(0.203)$ & $\mathrm{BP}(20)=14.21$ & $\mathrm{~J} 13=1.229$ \\
$K L_{t}$ & $\operatorname{IMA}(1,1)$ & $-0.921(0.017)$ & $\mathrm{BP}(20)=15.89$ & $\mathrm{JB}=14.55^{*}$ \\
\hline
\end{tabular}

* Significant at a $1 \%$ level.

a The models are $\phi(B) \Delta_{1} Z_{t}=\epsilon_{t}+\theta \epsilon_{t-1}$, where $\phi(B)$ is the autoregressive polynomial. For all variables, except $D 66_{t}$, this $\phi(B)=1$. For $D 66_{t}$ the $\phi(B)$ includes terms up to $B^{7}$.

b The estimate of the $\theta$ parameter in the MA(1) part of the models.

c The Box-Pierce test for residual autocorrelation up to lag 20 , distributed as $\chi^{2}(20-k)$, where $k$ is the number of ARMA terms in the model. The Jarque-Bera test checks for residual normality, and is $x^{2}(2)$ distributed under the null hypothesis.

2 we display the results of our univariate model building strategy. Except for the $D 66_{t}$ series, we find that $\operatorname{IMA}(1,1)$ models like

$$
Z_{t}-Z_{t-1}=\epsilon_{t}+\theta \epsilon_{t-1}
$$

are adequate data descriptions. The $\epsilon_{t}$ process in (1) is assumed to be a zero mean uncorrelatcd process with constant variance, i.e., a standard white noise process. For the $D 66_{t}$ series we obtain an ARIMA $(7,1,1)$ model. The adequacy of the various models is checked using the Box-Pierce test for the significance of the first 20 residual autocorrelations and the Jarque-Bera test for residual normality. All models do not show residual correlation, but for four of the six models normality can be rejected. One may now proceed with the inclusion of a few dummy variables to remove outlying observations, but we do not pursue this strategy. The only conclusion we draw from the nonnormal errors is that the estimated standard errors for the MA(1) parameters $\theta$, reported in the fourth column of Table 2 , should be treated with care.

The noticeable aspect from the univariate results in Table 2 is that the estimates for $\theta$ in (1) are typically close, although not equal to, -1 . These high values indicate that the processes have slowly decaying autocorrelation patterns, see Wichern (1973), which was also observed in Table 1. Furthermore, although we do not formally test for it, it is unlikely that the $\theta$ values are exactly equal to -1 . This is because the model (1) then would become $Z_{t}=\epsilon_{t}$, i.e., $Z_{t}$ is a white noise process, since the $(1-B)$ filter cancels out from both sides. Given the autocorrelations in Table 1, this does not seem to be the case for the six time series at hand. 


\section{Econometric issues}

This section discusses several econometric issues. We first consider the measurement of convergence. Next, we focus on the analysis of nonstationary time series in a multivariate model, and on the implications of such a model for the univariate time series. Finally, we propose a procedure to test for the convergence hypothesis which uses tests for trend stationarity in a multivariate time series model.

\section{Convergence}

The issue of convergence has stimulated much research in recent years in empirical economics. The possible usefulness of the European Monetary System, for example, depends on the convergence of financial measures of several European countries. Furthermore, the convergence of developing and industrialized countries is a topic of ample investigation.

The obvious question with respect to convergencc is how to measure it. In fact, one can typically consider two closely related hypotheses, i.e., whether a system has converged or whether it is converging. The first hypothesis involves an investigation of the properties of the system in the beginning and at the end of a sample period. These properties can be derived from qualities as stability, i.e., whether at the end of the period the system is more stable in some sense, or from forecast intervals, i.e., whether forecasts initially are less precise and get more precise as time proceeds. In our case, however, it seems more interesting to test the hypothesis whether the variables are converging.

If one has two variables, say $X_{t}$ and $Y_{t}$, which are measured at time $t$, where $t=1,2, \ldots, T$, one can say that these variables show a converging pattern if $\left(X_{t} / Y_{t}\right) \rightarrow 1$ when $T \rightarrow \infty$. Taking natural logarithms on both sides, this means that $\log \left(X_{t} / Y_{t}\right) \rightarrow 0$, when $T \rightarrow \infty$. Assuming that the starting values for $X_{t}$ exceed those of $Y_{t}$, a simple test for converging patterns amounts to checking the significance of the deterministic trend term $t$ in the model

$$
\phi(B)\left[\log \left(X_{t} / Y_{t}\right)-\alpha_{0}-\alpha_{1} t\right]=\epsilon_{t},
$$

where the sign of $\alpha_{1}$ should be negative. The $\alpha_{0}$ and $\alpha_{1}$ are unknown parameters and $\phi(B)$ is a polynomial in $B$. Hence, we assume that $\phi(B)$ can be written as $\phi(B)=1-\phi_{1} B-\phi_{2} B^{2}-\cdots-\phi_{p} B^{p}$. Finally, we assume that all roots of $\phi(B)$ lie outside the unit circle. If one of the roots is on the unit circle, then, loosely speaking, the process $\log \left(X_{t} / Y_{t}\right)$ does not revert to its 
deterministic trend pattern, and hence there is no convergence. We will get back to this issue in the discussion of unit roots and cointegration.

Of course, this approach can only be applied when the time series $X_{t}$ and $Y_{t}$ have not yet converged. In fact, when they have converged, the significance of $\alpha_{1}$ in (2) indicates that eventually the processes will diverge. Hence, (2) is only useful when the processes are converging. Furthermore, note that (2) implies that the difference between $\log X_{t}$ and $\log Y_{t}$ is a trend-stationary process since we do not need the $\Delta_{1}$ filter to remove the nonstationarity in $\log \left(X_{t} / Y_{t}\right)$. To simplify notation in the sequel of this section, we use $x_{t}=$ $\log X_{t}$ and $y_{t}=\log Y_{t}$.

\section{Multivariate time series and stationarity}

If one has two time series $x_{t}$ and $y_{t}$, one may consider a multivariate time series model for the $(2 \times 1)$ vector process $\left(x_{t}, y_{i}\right)^{\prime}$. For expositional convenience we assume that such a multivariate model is given by the following first order vector autoregression [VAR]:

$$
\left[\begin{array}{l}
x_{t} \\
y_{t}
\end{array}\right]=\left[\begin{array}{ll}
\beta_{1} & \beta_{2} \\
\beta_{3} & \beta_{4}
\end{array}\right]\left[\begin{array}{l}
x_{t-1} \\
y_{t-1}
\end{array}\right]+\left[\begin{array}{l}
\epsilon_{1 t} \\
\epsilon_{2 t}
\end{array}\right]
$$

where we assume that $\epsilon_{1 t}$ and $\epsilon_{2 t}$ are two independent white noise processes, which implies that $\epsilon_{1 t}$ and $\epsilon_{2 t-j}$ are uncorrelated for any $j=0, \pm 1, \pm 2, \ldots$ The variances of $\epsilon_{1 t}$ and $\epsilon_{2 t}$ are denoted by $\sigma_{1}^{2}$ and $\sigma_{2}^{2}$. Using the backward shift operator $B$, we rewrite (3) as

$$
\left[\begin{array}{cc}
1-\beta_{1} B & -\beta_{2} B \\
-\beta_{3} B & 1-\beta_{4} B
\end{array}\right]\left[\begin{array}{l}
x_{t} \\
y_{t}
\end{array}\right]=\left[\begin{array}{c}
\epsilon_{1 t} \\
\epsilon_{2 t}
\end{array}\right]
$$

where the $(2 \times 2)$ matrix on the left-hand side is labelled as $\beta(B)$ below.

The process $\left(x_{t}, y_{t}\right)^{\prime}$ is stationary if the solutions to $|\beta(\xi)|=0$, i.e.,

$$
1-\left(\beta_{1}+\beta_{4}\right) \xi+\left(\beta_{1} \beta_{4}-\beta_{2} \beta_{3}\right) \xi^{2}=0
$$

lie outside the unit circle. When one solution to (5) is on the unit circle, there is one unit root in the multivariate system. A single unit root emerges when $\beta_{1}+\beta_{4}-\beta_{1} \beta_{4}+\beta_{2} \beta_{3}=1$, i.e., when $\beta_{2} \beta_{3}=\left(1-\beta_{1}\right)\left(1-\beta_{4}\right)$, for example. Two unit roots emerge, for example, when $\beta_{1}=\beta_{4}=1$ and $\beta_{2}=$ $\beta_{3}=0$ since (5) then becomes $(1-\xi)^{2}$. 


\section{Implied univariate time series models}

Given the adequacy of a multivariate model like (3), it is possible to derive the implied univariate time series models for $x_{t}$ and $y_{t}$, see Granger and Newbold (1986). This can be done by recognizing that $(\beta(B))^{-1}=$ $\beta *(B) /|\beta(B)|$, with $\beta^{*}(B)$ denoting the adjoint matrix of $\beta(B)$. For the bivariate case in (3) this amounts to

$$
\begin{aligned}
{\left[\begin{array}{cc}
1-\beta_{1} B & -\beta_{2} B \\
-\beta_{3} B & 1-\beta_{4} B
\end{array}\right]^{-1}=} & 1 /\left[1-\left(\beta_{1}+\beta_{4}\right) B+\left(\beta_{1} \beta_{4}-\beta_{2} \beta_{3}\right) B^{2}\right] \\
& \times\left[\begin{array}{cc}
1-\beta_{4} B & \beta_{2} B \\
\beta_{3} B & 1-\beta_{1} B
\end{array}\right] .
\end{aligned}
$$

Premultiplying (4) on both sides with (6), and then multiplying both sides by $|\beta(B)|$ gives

$$
\begin{gathered}
{\left[1-\left(\beta_{1}+\beta_{4}\right) B+\left(\beta_{1} \beta_{4}-\beta_{2} \beta_{3}\right) B^{2}\right]\left[\begin{array}{l}
x_{t} \\
y_{t}
\end{array}\right]} \\
\quad=\left[\begin{array}{cc}
1-\beta_{4} B & \beta_{2} B \\
\beta_{3} B & 1-\beta_{1} B
\end{array}\right]\left[\begin{array}{l}
\epsilon_{1 t} \\
\epsilon_{2 t}
\end{array}\right],
\end{gathered}
$$

i.e., $\operatorname{ARMA}(2,1)$ processes for the univariate $x_{t}$ and $y_{t}$ series. When there is a single unit root in the multivariate system, these models become $\operatorname{ARIMA}(1,1,1)$ because $1-\left(\beta_{1}+\beta_{4}\right) B+\left(\beta_{1} \beta_{4}-\beta_{2} \beta_{3}\right) B^{2}$ can be written as $(1-B)\left(1-\left(\beta_{1}+\beta_{4}-1\right) B\right)$.

As a simple example, consider the following additional assumptions on the error processes: $\sigma_{1}^{2}=\sigma_{2}^{2}=1$, and write the $\mathrm{MA}(1)$ processes on the right-hand side of (7) as $\left(1-\theta_{z} B\right) \psi_{z t}$, where $z=x$ or $y$ and $\psi_{z t}$ is again a standard white noise process. It is not difficult to observe from (7) that the first order autocorrelation of $\psi_{z t}$ is $-\beta_{4} /\left(1+\beta_{4}^{2}+\beta_{2}^{2}\right)$ and $-\beta_{1} /\left(1+\beta_{1}^{2}+\beta_{3}^{2}\right)$, respectively. When we set, for example, $\beta_{1}=\beta_{4}=0.9$ and $\beta_{2}=\beta_{3}=0.1$, it is easy to derive that the two models are approximately $(1-0.8 B) \Delta_{1} z_{t}=(1-0.86 B) \psi_{z t}$, where $z_{t}$ is either $x_{t}$ or $y_{t}$. Notice that the MA(1) component of these models bears similarities with the models we estimated in the previous section, in the sense that the $\theta$ parameter is quite close to -1 . 


\section{Cointegration}

A natural procedure after finding univariate $\mathrm{I}(1)$ processes, i.e., processes that have to be filtered using $\Delta_{1}$ to obtain stationarity, is to construct models for multiple time series where one includes only the first differenced time series. For our example in (3), which we can write as

$$
\left[\begin{array}{c}
\Delta_{1} x_{t} \\
\Delta_{1} y_{t}
\end{array}\right]=\left[\begin{array}{cc}
\beta_{1}-1 & \beta_{2} \\
\beta_{3} & \beta_{4}-1
\end{array}\right]\left[\begin{array}{l}
x_{t-1} \\
y_{t-1}
\end{array}\right]+\left[\begin{array}{c}
\epsilon_{1 t} \\
\epsilon_{2 t}
\end{array}\right],
$$

this approach amounts to restricting (8) to

$$
\left[\begin{array}{c}
\Delta_{1} x_{t} \\
\Delta_{1} y_{t}
\end{array}\right]=\left[\begin{array}{c}
\epsilon_{1 t} \\
\epsilon_{2 t}
\end{array}\right]
$$

When we denote the $(2 \times 2)$ matrix on the right-hand side of $(8)$ as $\Pi$, the model in (9) corresponds to $\operatorname{rank}(\Pi)=0$. In other words, we have reduced the model in (8) via a reduction of the rank of $\Pi$. When the rank of $\Pi$ is equal to 2 , there are no possibilities to reduce the rank of $\Pi$. This implies that each of the univariate series $x_{t}$ and $y_{t}$ is stationary. However, there is an intermediate case, i.e., when the rank of $\Pi$ is equal to 1 . This is the case when, e.g., the restriction $\beta_{2} \beta_{3}=\left(1-\beta_{1}\right)\left(1-\beta_{4}\right)$ holds since then

$$
\begin{aligned}
{\left[\begin{array}{cc}
\beta_{1}-1 & \beta_{2} \\
\beta_{3} & \beta_{4}-1
\end{array}\right] } & =\left[\begin{array}{cc}
\beta_{2} \beta_{3} /\left(\beta_{4}-1\right) & \beta_{2} \\
\beta_{3} & \beta_{4}-1
\end{array}\right] \\
& =\left[\begin{array}{c}
\beta_{2} \beta_{3} /\left(\beta_{4}-1\right) \\
\beta_{3}
\end{array}\right]\left[1,-\left(1-\beta_{4}\right) / \beta_{3}\right]
\end{aligned}
$$

i.e., $\Pi$ can be decomposed into $\alpha \beta^{\prime}$ whcre $\alpha$ and $\beta$ are $(2 \times 1)$ vectors. Given this decomposition in $(10)$, one can rewrite $(8)$ as

$$
\begin{aligned}
& \Delta_{1} x_{t}=\left(\beta_{2} \beta_{3} /\left(\beta_{4}-1\right)\right)\left[x_{t-1}-\left[\left(1-\beta_{4}\right) / \beta_{3}\right] y_{t-1}\right]+\epsilon_{1 t} \\
& \Delta_{1} y_{t}=\beta_{3}\left[x_{t-1}-\left[\left(1-\beta_{4}\right) / \beta_{3}\right] y_{t-1}\right]+\epsilon_{2 t},
\end{aligned}
$$

Since the error processes $\epsilon_{1 t}$ and $\epsilon_{2 t}$, and the $\Delta_{1}$ transformed time series are $\mathrm{I}(0)$, the variable $x_{t-1}-\left[\left(1-\beta_{4}\right) / \beta_{3}\right] y_{t-1}$ is $\mathrm{I}(0)$ as well. This variable is called the error correction variable. It is now said that $x_{t}$ and $y_{t}$ are cointegrated, see Engle and Granger (1987). The cointegrating vector is 
$\left(1,-\left(1-\beta_{4}\right) / \beta_{3}\right)$ and $\left(\beta_{2} \beta_{3} /\left(\beta_{4}-1\right)\right)$ and $\beta_{3}$ are the adjustment parameters. The model in (11) and (12) is called an error correction model.

When we consider our numerical example again, where we set $\beta_{1}=\beta_{4}=$ 0.9 and $\beta_{2}=\beta_{3}=0.1$, the models in (11) and (12) become

$$
\begin{aligned}
& \Delta_{1} x_{t}=-0.1\left(x_{t-1}-y_{t-1}\right)+\epsilon_{1 t} \\
& \Delta_{1} y_{t}=0.1\left(x_{t-1}-y_{t-1}\right)+\epsilon_{2 t} .
\end{aligned}
$$

Notice that this simple bivariate error correction model yields quite complicated univariate time series models, like $\operatorname{ARIMA}(1,1,1)$, as we have seen above. Hence, this example suggests that if one is interested in a multivariate analysis anyhow, then one may start with such an analysis straightaway.

Consider now the $(m \times 1)$ vector process $W_{t}$ and assume that it can be described by a VAR process of order $k$, i.e.,

$$
W_{t}=\Gamma_{1} W_{t-1}+\cdots+\Gamma_{k} W_{t-k}+\mu+v_{t}
$$

where $\Gamma_{1}$ through $\Gamma_{k}$ are $(m \times m)$ matrices, $\mu$ is an $(m \times 1)$ vector process containing intercept terms and $v_{t}$ is an $(m \times 1)$ vector white noise process. Analogous to (8), this process in (13) can be rewritten as

$$
\Delta_{1} W_{t}=\Pi_{1} \Delta_{1} W_{t-1}+\cdots+\Pi_{k-1} \Delta_{1} W_{t-(k-1)}+\Pi W_{t-k}+\mu+v_{t}
$$

where $\Pi_{1}$ through $\Pi_{k-1}$ and $\Pi$ are functions of the $\Gamma_{i}$ in (13), $i=1,2, \ldots, k$, and where $\Pi$ contains useful information on possible cointegration properties of the elements in $W_{t}$. When the rank of $\Pi$ equals $m$, the $W_{t}$ process is stationary. When the rank of $\Pi$ equals 0 , the model in (14) reduces to a model for first differenced time series only. When $0<\operatorname{rank} \Pi<m$, there is cointegration between the elements of $W_{t}$. In other words, a test procedure for the presence of cointegration may be based on an estimate of the rank of $\Pi$ in (14).

The cointegration testing method proposed in Johansen and Juselius (1990) considers the rank of $\Pi$. For this purpose, one has to rely on reduced rank regression. This amounts to the following computations. First, one regresses $\Delta_{1} W_{t}$ and $W_{t-k}$ on a constant and the $\Delta_{1} W_{t-1}$ through $\Delta_{1} W_{t-(k-1)}$ variables, giving the $(m \times 1)$ residual vector processes $r_{0 t}$ and $r_{1 t}$ and their $(m \times m)$ residual product matrices 


$$
S_{i j}=(1 / T) \sum_{t=1}^{T} r_{i t} r_{j t}^{\prime}, \quad \text { for } i, j=0,1
$$

The next step is to solve the eigenvalue problem

$$
\left|\lambda S_{11}-S_{10} S_{00}^{-1} S_{01}\right|=0
$$

which gives the eigenvalues $\lambda_{1}>\cdots>\lambda_{m}$ and the corresponding eigenvectors $\hat{v}_{1}$ through $\hat{v}_{m}$. A test for the rank of $\Pi$ amounts to testing whether the $\left(1-\lambda_{i}\right)$ are equal to unity. Hence, the vectors $v_{i}$ that correspond to the largest $\lambda_{i}$ may correspond to the most stationary relationships between the elements of $W_{t}$.

One of the tests for the rank of $\Pi$ is the so-called trace test statistic $\operatorname{Tr}[\operatorname{rank}(\Pi)=r]$, which is calculated as

$$
\operatorname{Tr}[\operatorname{rank}(\Pi)=r]=-T \sum_{i=r+1}^{m} \log \left(1-\hat{\lambda}_{i}\right)
$$

This test statistic is useful to test the hypothesis that the rank of $\Pi$ equals $r$ versus $r+1$. Hence, rejection implies that the rank may equal $r+1$. Critical values for this test statistic if the estimated model is (13), with only the constant term $\mu$ included, are tabulated in Johansen and Juselius (1990).

\section{Testing for convergence}

Now we return to our case of interest, where we wish to investigate whether the linear combinations of the log transformed time series are trend-stationary processes as in (2), prior to testing whether the relevant $\hat{\alpha}_{1}$ are significantly negative. A convenient approach to this problem is to construct the time series $\log \left(K R_{t} / D 66_{t}\right), \quad \log \left(V V D_{t} / D 66_{t}\right), \quad \log \left(C D A_{t} / D 66_{t}\right)$, $\log \left(P v d A / D 66_{t}\right)$ and $\log \left(K L_{t} / D 66_{t}\right)$, which constitute the $(5 \times 1)$ vector process $V_{t}$. Since we wish to allow for trend stationarity of each of these processes, we consider an extension of model (14), i.e.,

$$
\Delta_{1} V_{t}=\Pi_{1} \Delta_{1} V_{t-1}+\cdots+\Pi_{k-1} \Delta_{1} V_{t-(k-1)}+\Pi V_{t-k}+\delta t+\mu+v_{t}
$$

where $\delta$ is a $(5 \times 1)$ vector of parameters and $t$ is the deterministic trend.

Given that we want to allow for univariate trend-stationary time series, and do not want to consider quadratic trend-like patterns (which may appear 
when the rank of $\Pi$ in (18) equals 0 and $\delta \neq 0$ ), we have to modify the aforementioned calculation steps slightly. See Johansen (1994) for further details of the role of the constant and trend terms in cointegration analysis. A first step. is that one regresses $\Delta_{1} V_{t}$ and $\left(V_{t-k}, t\right)$ on a constant and the $\Delta_{1} V_{t-1}$ through $\Delta_{1} V_{t-(k-1)}$ variables, giving the $(5 \times 1)$ residual vector process $r_{0 t}^{*}$ and the $(6 \times 1)$ vector process $r_{1 t}^{*}$ and their residual product matrices

$$
S_{i j}^{*}=(1 / T) \sum_{i=1}^{T} r_{i t}^{*} r_{j t}^{* \prime}, \quad \text { for } i, j=0,1,
$$

where $S_{00}^{*}$ is a $(5 \times 5)$ matrix, $S_{01}^{*}$ is a $(6 \times 5)$ matrix and $S_{11}^{*}$ is a $(6 \times 6)$ matrix. The next step is to solve the eigenvalue problem

$$
\left|\lambda^{*} S_{11}^{*}-S_{10}^{*} S_{00}^{*-1} S_{01}^{*}\right|=0
$$

which gives the eigenvalues $\lambda_{1}^{*}>\cdot>\lambda_{5}^{*}>0$ and the corresponding eigenvectors $\hat{v}_{1}^{*}$ through $\hat{v}_{6}^{*}$.

The test for the rank of the $\Pi$ matrix in (18) is equal to that in (17), where the zero-valued eigenvalue is not included. The critical values of this trace test are tabulated in Johansen (1994, Table V, p. 227). There is convergence amongst our six ideological position series if we find that the rank of the $\Pi$ matrix is equal to 5 , i.e., when our $(5 \times 1)$ vector series $V_{t}$ is a trendstationary process. An analysis of the individual series using the model in (2) yields an indication as to which pairs of ideological positions series are converging more rapidly than others.

\section{Results}

In this section we report the empirical results obtained from estimating a $\operatorname{VAR}(k)$ process like $(18)$ for the $(5 \times 1)$ vector $V_{t}$, and from estimating models like (2) to the various linear combinations.

After some experimentation with lag lengths $k$ for the VAR process, we set $k$ equal to 4 . For this model order, the residual autocorrelations in the five model equations are not significant, while they get significant when $k$ is set equal to 3 or 2 . The results of the calculation of the eigenvalues $\lambda_{i}^{*}$ and 
Table 3. Application of the Johansen cointegration method to the $(5 \times 1)$ vector process containing $\log \left(K R_{t} / D 66_{t}\right), \log \left(V V D_{t} / D 66_{t}\right), \log \left(C D A_{t} / D 66_{t}\right), \log \left(P v d A / D 66_{t}\right)$ and $\log \left(K L_{t} / D 66_{t}\right)$. The estimation model is (18).

\begin{tabular}{lll}
\hline Eigenvalue & $\operatorname{Tr}[\operatorname{rank}(\Pi)=r$ versus $r+1]$ & $1 \%$ critical value \\
\hline$\lambda_{1}^{*}=0.200$ & $r=4$ vs. $r=5: 48.95^{* *}$ & 16.45 \\
$\lambda_{2}^{*}=0.164$ & $r=3$ vs. $r=4: 158.9^{* *}$ & 30.55 \\
$\lambda_{3}^{*}=0.149$ & $r=2$ vs. $r=3: 281.9^{* *}$ & 48.99 \\
$\lambda_{4}^{*}=0.135$ & $r=1$ vs. $r=2: 418.4^{* *}$ & 70.63 \\
$\lambda_{5}^{*}=0.062$ & $r=0$ vs. $r=1: 588.4^{* *}$ & 95.15 \\
\hline
\end{tabular}

** Significant at a $1 \%$ level. The critical values are given in Table V (p. 227) in Johansen (1994). The eigenvalues are calculated by solving (20). The trace test statistic is given in (17), The number of effective observations is 761 .

Table 4. Estimated parameters for linear trend in relative ideological position series.

\begin{tabular}{lccc}
\hline Relative position & Lag order $^{\mathrm{a}}$ & $\hat{\alpha}_{1}(\times 1000)$ & $t$ ratio \\
\hline $\log \left(K R_{t} / V V D_{t}\right)$ & 0 & -0.066 & $-8.587^{*}$ \\
$\log \left(K R_{t} / C D A_{t}\right)$ & 0 & 0.011 & 1.574 \\
$\log \left(K R_{t} / D 66_{t}\right)$ & 11 & -0.197 & $-9.404^{*}$ \\
$\log \left(K R_{t} / P v d A_{t}\right)$ & 10 & -0.297 & $-10.953^{*}$ \\
$\log \left(K R_{t} / K L_{t}\right)$ & 7 & -0.321 & -10.682 \\
$\log \left(V V D_{t} / C D A_{t}\right)$ & 1 & -0.055 & $-10.435^{*}$ \\
$\log \left(V V D_{t} / D 66_{t}\right)$ & 3 & -0.137 & $-12.628^{*}$ \\
$\log \left(V V D_{t} / P v d A_{t}\right)$ & 7 & -0.234 & $-10.057^{*}$ \\
$\log \left(V V D_{t} / K L_{t}\right)$ & 7 & -0.264 & $-9.094^{*}$ \\
$\log \left(C D A_{t} / D 66_{t}\right)$ & 3 & -0.192 & $-18.051^{*}$ \\
$\log \left(C D A_{t} / P v d A_{t}\right)$ & 9 & -0.283 & $-11.831^{*}$ \\
$\log \left(C D A_{t} / K L_{t}\right)$ & 7 & -0.317 & $-11.663^{*}$ \\
$\log \left(D 66_{t} / P v d A_{t}\right)$ & 10 & -0.107 & $-4.176^{*}$ \\
$\log \left(D 66_{t} / K L_{t}\right)$ & 9 & -0.133 & $-4.921^{*}$ \\
$\log \left(P v d A_{t} / K L_{t}\right)$ & 6 & -0.031 & -1.454 \\
\hline
\end{tabular}

* Significant at a $5 \%$ level.

a The estimated model is (2), where the lag order is $p$. Number of effective observations in each regression is $765-p$.

the trace test statistics in (17) are displayed in Table 3. Comparing the trace test results with the $1 \%$ critical values, we conclude that any rank reduction of $\Pi$ is rejected, and hence that $V_{t}$ seems a trend-stationary process. Notice that when $\log \left(X_{t} / Y_{t}\right)$ and $\log \left(Z_{t} / Y_{t}\right)$ are $\mathrm{I}(0)$ stationary, that $\log \left(X_{t} / Z_{t}\right)$ is $\mathrm{I}(0)$ too.

The next step is to examine whether the six ideological positions converge by checking whether the estimates for $\alpha_{1}$ in (2) are significantly negative for the $\log \left(X_{t} / Y_{t}\right)$ process when $X_{t}$ usually exceeds $Y_{t}$. The relevant estimation results for all pairs are displayed in Table 4 . It can be observed that for 13 of the 15 pairs the $\hat{\alpha}_{1}$ is negative and significant. For two pairs, i.e., 
$\left(K R_{t}, C D A_{t}\right)$ and $\left(P v d A_{t}, K L_{t}\right)$, there appears to be no significant convergence. Table 4 also shows that the larger the differences in level between the series, the faster the pairs converge. Series with relatively low and high values on the left-right continuum, e.g., the pairs $\left(K R_{t}, K L_{t}\right)$ and $\left(C D A_{t}, K L_{t}\right)$ converge relatively fast, while pairs with more or less corresponding levels, e.g., $\left(V V D_{t}, C D A_{t}\right)$, converge relatively slow.

\section{Concluding remarks}

This paper uses the Johansen and Juselius (1990) cointegration approach to show how convergence may be dealt with effectively when variables follow linear stochastic processes that are stationary in first differences rather than in levels. The method was applied to left-right ideological orientation in the Netherlands. It was found that the hypothesis of convergence, at least in the variant considered here, cannot be rejected. This finding can be interpreted as evidence of the assumption that the relevance of the left-right continuum as a generalized way of reference for political and ideological thinking is declining. The implication of this finding is that the Dutch voting population is gradually becoming more homogeneous in its ideological orientation. This process of de-ideologisation may ultimately result in an 'end-of-ideology'. Finally, from a pure practical perspective, the fact that simple multivariate error correction models can yield complicated univariate time series models suggests that researchers interested in a multivariate model may restrict themselves to a multivariate analysis.

\section{Acknowledgement}

Both authors thank the Royal Netherlands Academy of Arts and Sciences for its financial support.

\section{References}

Box, G. E. P. and Jenkins, G. M. (1970). Time Series Analysis, Forecasting and Control, San Francisco: Holden-Day.

Eisinga, R. and Felling, A. (1992). Confessional and Electoral Alignments in the Netherlands. Documentation of Social Background Variables of 1,067 National Surveys Conducted by NIPO from 1962 to 1992, Amsterdam: Steinmetz Archive.

Engle, R. F. and Granger, C. W. J. (1987). Co-integration and error correction: Representation, estimation, and testing, Econometrica 55: 251-276. 
Granger, C. W. J. and Hallman, J. (1991). Nonlinear transformations of integrated time series, Journal of Time Series Analysis 12: 207-224.

Granger, C. W. J. and Newbold, P. (1986). Forecasting Economic Time Series, San Diego: Academic Press.

Johansen, S. (1994). The role of the constant and linear terms in cointegration analysis of nonstationary variables, Econometric Reviews 13: 205-229.

Johansen, S. and Juselius, K. (1990). Maximum likelihood estimation and inference on cointegration - with applications to the demand for money, Oxford Bulletin of Economics and Statistics 52: 169-210.

Wichern, D. (1973). The behaviour of the sample autocorrelation function for an integrated moving average process, Biometrika 60: 235-239. 